\title{
Flow over cube arrays of different packing densities
}

\author{
H. Cheng, P. Hayden, A.G. Robins and I.P. Castro* \\ EnFlo, School of Engineering, University of Surrey, Guildford, GU2 7XH, U. K. \\ * School of Engineering Sciences, University of Southampton, Southampton, SO17 1BJ, U. K.
}

\begin{abstract}
Measurements by $120^{\circ}$ x-wire anemometry over uniform urban-type surfaces of two different area densities were performed in a wind tunnel. The aerodynamic characteristics for each surface were determined and compared and the influence of area density on these parameters was examined. Various approaches to determine the roughness length $\left(z_{0}\right)$ for a given surface are discussed. The surface shear stress (determined from form drag measurements by pressure tapping a roughness element), the total surface drag (determined by a floating drag balance), and the stress (determined from measured vertical profile of Reynolds shear stress over the roughness) were compared. It was found that the surface shear stress is about $25 \%$ greater than the measured Reynolds shear stress in the inertial sublayer over the surfaces. It is also revealed that the centroid height of the drag force, which is a function of the surface geometry and relatively independent of the flow, increases with increasing area coverage density; it seems likely that it is coincident with the zero-plane displacement for the surface with low area density for which the flow has not started to skim the roughness elements.
\end{abstract}

Keywords: Urban roughness, drag force, area density, roughness length and wind tunnel.

\section{Introduction}

The estimation of surface characteristics is needed by many meteorological and windengineering applications concerned with air pollution and environmental wind effects. The overall effect of the surface is often represented by the roughness length $z_{0}$ and/or the surface friction velocity $u^{*}$, both of which describe the roughness of the surface. In dispersion modelling, these are both important and essential input parameters. Accurate knowledge of the aerodynamic characteristics of rough surfaces is vital to model and forecast the behaviour of urban wind and turbulence as well as urban air quality.

As pointed out by Grimmond and Oke [1], there are generally two different approaches to determining the aerodynamic characteristics for a given rough surface. One is a geometrical method, which determines the aerodynamic parameters from the surface geometry. This method is based on empirical relations derived from wind tunnel simulations over idealised surfaces. The second is a micrometeorological method, which uses direct field observation of wind and turbulence to deduce the roughness properties by assuming logarithmic wind profiles. The former is generally more widely used than the latter due to the high cost associated with operating experiments in the field. However the "actual" or "true" surface characteristics can only be estimated from wind profile measurements taken over the surface [2] and a reliable evaluation of $z_{0}$ and the zero-plane displacement $d$ requires the existence of a logarithmic layer in the flow over the rough surface [3]. Because of the height limitation of measurement towers, meteorological data are often obtained in the roughness sublayer, which is likely to be 
inappropriate for overall parameterisation. Therefore, the understanding of boundary layer meteorology leans heavily on experimental measurements in wind tunnels.

Grimmond and Oke [1] also conducted sensitivity analyses on several morphometric methods of determining $z_{0}$ and $d$, and made comparison with values obtained from wind observations, but they still could not make a definitive judgement or provide recommendations concerning which methods should be used, because of the lack of high quality measurements over urban roughness. After carefully screening the available data from the literature, they pointed out that there are few credible estimations of $z_{0}$ and almost none of $d$, therefore there is no credible standard against which to validate morphometric formulae.

In the early studies of determining surface characteristics, the most common problem was failure to include $d$ in the analysis $-d$ was often (inappropriately) assumed to be zero [1]. For rough surfaces with very low density, where the zero-plane displacement may well be very small, this assumption probably has little effect on the determination of the roughness length. But for practical urban surfaces, the density is often relatively high. Spanton et al. [4] surveyed some typical UK urban regions and found area densities as high as 50-60\% in centrally situated commercial areas, while some industrial regions had area densities in the range of $20-40 \%$. In such cases, the value of $d$ may be a significant fraction of average building height, so the assumption of $d=0$ could lead to significant error in estimating the value of $z_{0}$. For example, in our early study for staggered cube arrays of $25 \%$ area coverage it was found that $d / h=0.83$ and $z_{0} / h=0.053$, where $h$ is the height of the roughness elements. If $d$ was forced to be zero, the loglaw fitting process would lead to $z_{0} / h=0.106$, which is $100 \%$ higher than it should be. The same behaviour was also observed by Bottema [3].

The main problem which causes the inaccuracy of determining $z_{0}$ is due to the fact that there is not much information about the depth of the roughness sublayer for a given surface and consequently inappropriate wind velocity data are often used to fit the log-law. Furthermore, measurements are often conducted at a location that did not have enough fetch for the flow to become fully developed, so that a unique inertial sublayer for the underlying surface does not exist. In that case, a log-law region is often inappropriately assumed in order to determine the surface properties (e.g. Macdonald et al., [5]), which leads to incorrect surface parameterisation. In addition, Counihan [6] observed that the roughness length is a strong function of fetch especially in the region before the flow becomes fully developed; this has been confirmed in our early work [7]. It is generally accepted that the surface layer over roughness can be divided into the inertial sublayer and roughness sublayer. At the top of the roughness sublayer, turbulent mixing smears individual wakes sufficiently to cause the flow to become independent of horizontal position and the flow "sees" the rough surface as uniformly rough and its detailed structure is unimportant. This height represents the minimum elevation above a rough surface at which the vertical profiles at an individual location are representative of the flow over the roughness.

The top of the roughness sublayer is often thought to be about 2-5h depending on the height and the spatial arrangement of the elements [8], but the precise depth is unknown over most surfaces and no systematic studies are available in the literature. Information on the depth of the roughness sublayer is very limited and confusing. O'Loughlin and Annambhotla [9] examined water channel flow over cube roughened boundaries of low roughness concentration, and suggested that the outer limit of the roughness sublayer occurs at $z_{r}=2 h$. Mulhearn and 
Finnigan [10], relying on wind tunnel experiments over random rough gravel surface, suggested that $z_{r}=2 \bar{D}$ where $\bar{D}$ is the average spacing between the roughness elements. Raupach et al. [11] studied regular cylinder arrays with five difference densities and suggested that $z_{r}=h+1.5 B$ (where $B$ is the inter-element spacing), without indicating the influence of the area density on the depth of the roughness sublayer. From our recent study, it has been found that the depth of the roughness sublayer, which is essentially independent of fetch, is $1.8 \mathrm{~h}$ for regular cube arrays and $2.5 \mathrm{~h}$ for a particular random height surface, even though all the surfaces have the same area density of $25 \%$ [7]; it is $4 h$ for uniform two dimensional square bar rib-type roughness and $5 h$ for vertical flat plates [12], where $h$ is the mean height of the roughness elements. The few expressions on the depth of the roughness sublayer available from the literature are probably an indication of our current understanding on the roughness sublayer.

It is well accepted that there are three possible flow regimes over urban roughness [13]. In the isolated flow regime, the wake and the separation bubble behind the obstacle are fully developed with re-attachment occurring before the next element. The bulk of the flow is forcibly displaced up and over the obstacle, which causes acceleration or a jet, but once over it is able to expand again and decelerates accordingly. This flow region, disturbed because of the presence of the obstacle, is called the displacement zone. With increasing density, the roughness elements become close enough so that the wake behind an obstacle starts to interfere with that of the downstream obstacle, leading to a complicated flow pattern. If the density increases further, the flow begins to skim over the elements. It has been reported that the maximum value of roughness length is obtained at the start of the wake interference flow [14]. The depth of the roughness sublayer is probably associated with the height of the displacement zone. The size and shape of the wake shed by a building depend on its width and depth as well as its height and distance to surrounding structures. It seems likely that the depth of the roughness sublayer depends on the geometry of the rough surface, such as the shape and size of the obstacles, their layout and the consequent area density.

Some studies [1, 15, 8] have shown that the roughness length, expressed as a fraction of building height, depends on frontal area density and/or plan area density. The roughness length increases with increasing area density, until a point comes where adding new roughness elements merely serves to reduce the effective drag of those already present, due to mutual sheltering. At the extreme density of $100 \%$ the elements are so closely packed that a new smooth surface is formed. This behaviour produces a peak in $z_{0}$ at some intermediate density. Similarly, with increasing density, the zero-plane displacement $d$ starts to move upwards and eventually the packing becomes so dense that eddies have difficulty penetrating the interelement space and the flow skims, so the level of the element roof becomes the new displacement height for the mean flow. Bottema [3] presented an analytical model for evaluation of $z_{0}$ and $d$ for regular building groups, and suggested that apart from the frontal density, the stream-wise building length and the building layout pattern are also important parameters.

There also exists a great deal of confusion about the zero-plane displacement $d$ in the literature. $z_{0}$ is regarded as the basic measure of the degree of surface roughness and by far the more significant parameter than $d$. In atmospheric measurements, one typically does not know $u^{*}, z_{o}$ and $d$, so all three unknowns have to be found from the log-law fitting of a velocity profile at a few heights. To reduce the uncertainty in this fitting process, $d$ is often assumed or approximated by the investigators, as in the work of Macdonald et al. [5, 15], which in turn 
contributes to the variability in evaluations of $z_{0}$ even for the same type of roughness. In more advanced studies, which are usually wind tunnel simulations, $u^{*}$ is independently determined from the vertical profiles of the Reynolds shear stress measurements. Even so, there still exists uncertainty in choosing which region of the velocity profile to fit by the log-law.

In all the cases mentioned above $d$ is no more than a fitting parameter. However, Jackson [16] argued that the displacement height in the log-law should be regarded as the level at which the mean drag on the surface acts. He further suggested that $d$ is determined by the distribution of the force on the surface, whereas the roughness length is determined by the magnitude of this force. Jackson's method is intuitively appealing, not only because $d$ is determined independently of the log-law fitting, but also because he gives $d$ a physical meaning. Most recently Kastner-Klein and Rotach [17] performed measurements at a few locations in the centreline of a long street over a complicated urban model. Because of the long and deep urban street canyon nature of their model, they observed negligible momentum flux at the lower section of the street and pronounced maxima above roof level in their shear stress profiles. They then introduced a shear stress displacement height $d_{s}$ and a length scale $z_{s}$, which is the height at which the maximum shear stress occurs. Based on their limited measurements, they formulated mathematical expressions for $d_{s}, z_{s}$ (as a function of plan area density) and shear stress profile (as a function of height) by quasi-physical arguments and curve fitting. By defining the zero-plane displacement $d$ as the level of mean momentum absorption [16] and relating their roughness length to $\left(z_{s}-d_{s}\right)$, they pointed out that both $z_{0}$ and $d$ can be expressed by linear correlations as a function of distance between the level of shear stress peak and the shear stress displacement height $\left(z_{s}-d_{s}\right)$. It is remarkable that by establishing the variation of the plan area density over a small area of complicated urban model they could then derive the roughness length for each individual vertical velocity profile; it is not clear that this would always be the best approach.

In addition, a recent review by Jiménez [18] has reiterated the importance of the ratio $\delta / h$ in understanding turbulent flow over rough walls ( $\delta$ and $h$ are the boundary layer thickness and the height of the roughness element respectively). Most of his review discussed the case of large $\delta / h$ but he pointed out that, for flows with small $\delta / h$, few of the mechanisms of normal classical wall turbulence remain and the influence of the roughness expands well beyond the near-wall region, if not across the whole boundary layer. For practical urban meteorological flows, the values of $\delta / h$ may well not be large. It is necessary to investigate flows in such cases in order to understand how pollutants are dispersed and transported in urban streets.

In this paper we will describe experimental results over uniform cube arrays of two different area densities in a wind tunnel to examine the influence of area density on surface characteristics at a blockage ratio $(\delta / h)$ of $6 \sim 7$. Surface shear stress, determined from form drag measurements by tapping a roughness element and total surface drag by a floating drag balance, are compared with the Reynolds shear stress in the inertial sublayer over the surface measured by a $120^{\circ} \mathrm{x}$-wire anemometry. We will compare the values of roughness length using the same range of the wind profile data for a given surface with different $u^{*}$, and test whether the centroid height at which the mean drag force acts is the same as the zero-plane displacement. The aim of this study is to investigate the effects of area density on the aerodynamic characteristics over urban-like roughness. This is a continuation of the work described in reference [7]. 


\section{Experiments arrangement}

The experiments were conducted in the EnFlo "A" tunnel, which has a test section $4.5 \mathrm{~m}$ long $0.6 \mathrm{~m}$ high and $0.9 \mathrm{~m}$ wide. The free stream velocity in the wind tunnel was measured by a Pitot static tube. $(x, y, z)$ are the stream-wise, lateral and vertical coordinates, with the plane $z=0$ being the top surface of the baseboard on which the roughness elements were located. Time and space averaged mean and fluctuating velocities will be denoted as $(U, V, W)$ and $(u$, $\left.v^{\prime}, w^{\prime}\right)$ respectively. Over-bars denote time averages and angle brackets denote spatial averages.

In this study, two area coverage densities (the ratio of the plan area occupied by the roughness elements to the total surface area) of $25 \%$ and $6.25 \%$ were used with four rough surfaces, with the cubes in staggered and aligned patterns as shown in Figure 1, where the area surrounded by the dashed line is a repeating unit and "+" denotes the locations of the vertical profiles. The roughness elements for all the surfaces were sharp edged wooden cubes of side $20 \mathrm{~mm}$. In order to prevent interference of the boundary layer from the upstream contraction of the wind tunnel, the rough surfaces were raised vertically by $60 \mathrm{~mm}$ above the tunnel floor and a section of aluminium flat plate with rounded leading edge was positioned at the front of the rough surface. In each case the roughness covers the entire length of the tunnel floor. All the experiments described here were performed at the tunnel centreline and about $3 \mathrm{~m}(x / h=150)$ downstream of the roughness leading edge.

The vertical profiles of mean flow and turbulence were measured by $120^{\circ}$ cross hot-wire anemometry, with corrections for high turbulence intensity [19], at a free stream velocity of 10 $\mathrm{m} / \mathrm{s}$. Form drag measurements were performed at free stream velocities of $4-10 \mathrm{~m} / \mathrm{s}$ by replacing one wooden cube with a brass one which was fitted with 21 pressure tappings located in different positions on the front and back of the cube. Pressure distributions at 42 locations on the front and back-side of the cube, as illustrated in Figure 2, were obtained by rotating the brass cube through $180^{\circ}$ and using a micro-manometer and a scani-valve. The static pressure from the Pitot-static tube, which was located in the free-stream above the measurement position, was used as the reference pressure. A sampling frequency of $200 \mathrm{~Hz}$ and a sampling time of about one minute at each position was generally used for both $x$-wire and form drag measurements.

In addition, the total surface drag was also measured by a floating drag balance, which can be briefly described as follows. Roughness element cubes (18 for $25 \%$ surfaces and 7 for the $6.25 \%$ surfaces) were glued onto a foam sheet as shown in Figure 3 in exactly the same pattern as the cube surfaces to form a raft. An oil bath, in which the raft was placed, was located at the tunnel centreline at about $3 \mathrm{~m}$ downstream of the leading edge of the roughness; its plan area is larger than the raft, and there was a $20 \mathrm{~mm}$ gap between the sides of the raft and the edges of the bath. The buoyancy of the raft was designed so that the top of the foam surface was just above the oil level, and the bath filled in order that the height of the cubes on the raft matched the surrounding array. Oil was chosen as the working fluid in the bath as its high viscosity is useful for damping oscillation of the raft in the experiments. A vertical tube (acting as a cantilever spring), to which the raft was tethered, was held by the overhead traversing system whose longitudinal position was adjusted to maintain the raft in the correct position relative to the surrounding arrays. This arrangement enabled the aerodynamic drag force acting on the cubes and raft surface to be obtained by measuring the cantilever deflection (via the change in traverse position) over a range of tunnel speeds. The stiffness of the cantilever spring was 
calibrated before the experiments and compensation was made for the force acting on the spring surface itself.

\section{Form drag measurements}

The drag force $D$ of the flow on an individual cube was determined by integrating the pressure difference between the front and back faces of the cube at corresponding positions over the frontal area.

$$
D=\int_{A}\left(p_{f}-p_{b}\right) d A
$$

where $p_{f}$ and $p_{b}$ are the pressures on the front and back faces respectively and $A$ is the frontal area of the roughness element. Area divisions used for the above integration are also shown in Figure 2. Further analysis by extrapolating and interpolating pressure values at the edge of the cube and inter-tapping positions based on the existing pressure measurements on the cube faces with a higher resolution in area divisions, not shown here, has confirmed that the present technique was adequate.

Based on the free stream velocity, the drag coefficient of an individual cube is expressed as

$$
C_{d}^{r}=\frac{2 D}{\rho U_{r}^{2} A}
$$

The surface shear stress over the repeating unit is given by

$$
\tau_{p}=\frac{D}{A_{c}}
$$

By neglecting the viscous drag on the repeating unit, the friction velocity of the surface is defined as

$$
u^{*}(p)=\sqrt{\frac{\tau_{p}}{\rho}}
$$

From the above equations the relationship between the drag coefficient of an individual roughness element and the friction velocity of the rough surface can be established as

$$
\frac{u^{*}(p)}{U_{r}}=\left(\frac{C_{d}^{r} \lambda_{f}}{2}\right)^{0.5}
$$

where $U_{r}$ is the free stream velocity, $A_{c}$ is the unit plane area of the roughness surface, $\tau_{p}$ is the shear stress due to the form drag, $u_{(p)}^{*}$ is the friction velocity determined from the form drag, $\rho$ is the air density and $\lambda_{f}=\frac{A}{A_{c}} . \quad C_{d}^{r}$ is the cube drag coefficient based on the free stream velocity. If the drag coefficient is based on the friction velocity, the expression $C_{d}^{*}=\frac{2}{\lambda_{f}}$ can be derived through re-arrangement of equations (2) to (4). This drag coefficient is only a function of the frontal area density, and is therefore 32 and 8 for the rough surfaces with area density of $6.25 \%$ and $25 \%$ respectively.

The results for $C_{d}^{r}$ and $u^{*}{ }_{(p)} / U_{r}$ versus free stream velocity are displayed in Figure 4(a) and (b) respectively. Both drag coefficient and the friction velocity are only a weak function of free stream velocity (or Reynolds number) in the conditions investigated. These results also showed 
that the staggered arrays generally produce higher resistance to the flow than the aligned cube arrays, and this is more evident at lower than higher area coverage, as illustrated in Figure 4(c). The drag coefficient of an individual element in a cube array is significantly smaller than that of an isolated cube in a turbulent boundary layer (typical values can be found in reference [20]) due to the sheltering effects in the cube arrays.

With increasing surface area coverage density, the drag force experienced by individual roughness elements decreases, and so does the drag coefficient, which is likely due to the mutual sheltering effects at higher packing density. However comparing the data of $u^{*}{ }_{(p)} / U_{r}$ for the four rough surfaces, it is found that the ratio of the friction velocities for packing density of $6.25 \%$ and $25 \%$ is almost independent of the free stream velocity, and is about 1 for the staggered pattern and 0.94 for the aligned pattern as shown in Figure 4(d). For the staggered arrays, the surface stresses for both surfaces are almost identical even though the roughness element arrangement in one is four times denser than the other, and the flows over the $6.25 \%$ and $25 \%$ surfaces are in different flow regimes, as discussed later in this section. This may be a coincidence as the roughness length is expected to go through a maximum when the packing density increases from 0 to 1 .

By integrating the pressure distribution on the roughness element, the centroid of the drag force can be determined from the following expression:

$$
\int_{A} z\left(p_{f}-p_{b}\right) d A=D \cdot H_{c}
$$

where $H_{c}$ is the height at which the mean drag of the flow acts on the rough elements. The results normalised by cube height for all four surfaces are plotted against free stream velocity in Figure 5. It is evident that the centroid height of the pressure force acting on the roughness element is independent of both the magnitude and the direction of the free stream velocity and is only a function of area coverage density in the conditions investigated, which implies that in these cases $H_{c}$ is determined by the surface geometry rather than the flow.

As stated in Section 2, the pressure distributions on the roughness elements were obtained for all four surfaces at free stream velocities of 4-10 m/s. The results are plotted in Figure 6 nondimensionally in the form of contours of constant local pressure difference coefficient, $C_{p}$, defined as:

$$
C_{p}=\left(p_{f}-p_{b}\right) / \frac{1}{2} \rho U_{r}^{2}
$$

As for the drag coefficient shown in Figure 4(a), the influence of the free stream velocity on the pressure coefficient is insignificant; for each surface the $C_{p}$ distributions at different free stream velocities remains similar and only those at $10 \mathrm{~m} / \mathrm{s}$ are displayed in the figure. Comparing the corresponding cube arrays with different area densities, the pressure coefficients on the individual cubes for $6.25 \%$ surfaces have much higher values than those of the $25 \%$ surfaces, which is undoubtedly due to the greater shelter effects at higher density. For the $25 \%$ surface, whether the roughness elements are arranged in staggered or aligned pattern, the range of the $C_{p}$ values is quite similar; this is probably because the packing density is so high that the flow above cannot penetrate into the urban canopy layer regardless of the wind direction. For the 
$6.25 \%$ surfaces, the distribution of $C_{p}$ is very similar to the results of the pressure distribution on an isolated cube immersed in a boundary layer flow, see [21, 22].

The largest pressure difference was observed in the region around $z=16 \mathrm{~mm}$ for the $6.25 \%$ surfaces, whereas it is at the very top of the cube for the $25 \%$ surfaces. It is speculated that the high-pressure area on the cube moves upward as the density increases until it reaches the height of the cube where the flow begins to skim the roughness elements. It is possible that, for the 20 $\mathrm{mm}$ cube arrays, the flow has started to skim the roughness elements at an area density well below 25\%. These results also confirmed that the staggered cube arrays are experiencing higher drag force than the aligned pattern for the $6.25 \%$ surfaces (similarly for the $25 \%$ surfaces but with less pronounced effects), which is consistent with the discussions of drag coefficient mentioned above.

\section{Vertical profiles results}

\subsection{Depth of the roughness sublayer}

For each of the four rough surfaces investigated, 16 vertical profiles, uniformly distributed in a repeating unit as shown in Figure 1, were taken. The vertical profiles of each quantity are displayed in Figures 7-10 for C20S-25\%, C20A-25\%, C20S-6.25\% and C20A-6.25\% respectively. Here $\mathrm{C}, \mathrm{S}$ and A stand, respectively, for cube, staggered and aligned. Comparing these data among the four roughnesses, $\mathrm{C} 20 \mathrm{~A}-6.25 \%$ is an exceptional case. It is evident that the flow feels the presence of the surface details at least up to a height around two-thirds of the boundary layer thickness. The roughness elements were so sparsely spaced in a spanwise sense that the flow in the wide channel between the cubes remains separate from the flow over the cubes. This makes parameterisation from one single vertical profile impossible for the surface. Here it is noteworthy that the ratios of $\delta / h$ for all the four surfaces studied here are about $6 \sim 7-$ the height of the roughness element is a significantly large fraction of the total boundary layer depth. The results for C20A-6.25\% roughness have certainly confirmed the statement made by Jiménez [18] that the effects of the roughness extend across the boundary layer depth for flow with a low ratio of $\delta / h$. Whether the flow over such a surface will develop into a state where the effects of the roughness elements vanish at a certain height and the logarithmic layer is found at great fetch, as claimed by Jiménez [18], is beyond the scope of this study and remains to be answered. However, these results have demonstrated that the blockage ratio of $\delta / h$ is not the only parameter which controls whether an inertial sublayer exists. It seems to indicate that the layout pattern (or the inter elements distance in the case of C20A-6.25\%) has an important role to play in determining the depth of the roughness sublayer. The vertical profiles of spatially averaged shear stress over a repeating unit for all the surfaces involved here are displayed in Figure 11. Strictly speaking there is no constant shear stress layer for these roughnesses at the conditions investigated; a possible explanation is given in Section 4.4.

The roughness sublayer generally defined as the region where the flow is strongly influenced by individual roughness elements. In this study, the upper limit of the roughness sublayer is defined qualitatively as the height where the influence of the geometry of the surface disappears and all the vertical profiles within a repeating unit converge. Because of the low ratio of $\delta / h$ for the flows involved here, a logarithmic layer, in the sense of regular smooth wall or in the rough wall boundary layer with larger $\delta / h$ such as $\delta / h>80$ [18], may not exist at all. As an attempt to find a way to describe the flow over practical urban surfaces the depth of the inertial sublayer is 
tentatively defined by the region above the roughness sublayer within which the vertical variation in spatially averaged shear stress is confined to $5 \%$. These definitions are somewhat subjective but alternative definitions, which are examined and discussed in Section 4.4, will not affect the main conclusions. From Figure 7-10, it is clear that in the inertial sublayer the variation with profile location within the repeating unit is relatively small compare to that in the roughness sublayer. As pointed out by Feddersen et al. [23], there exist two definitions for the inertial sublayer, one is the quasi-constant flux layer used in our early work [7] and the other is the region over which the wind profile can be fitted by the log-law. In this paper, we will examine whether these two definitions lead to similar surface parameters for a given roughness. The thickness of the boundary layer at the measurement location was defined as the height at which the mean velocity was $99 \%$ of the free stream velocity. The depth of each layer for each surface is listed in Table I and also indicated in Figures 7-10. Apart from the exceptional case of $\mathrm{C} 20 \mathrm{~A}-6.25 \%$, these results indicate that the depth of the roughness sublayer is essentially independent of the area coverage density and the wind direction for the conditions investigated here.

\subsection{Surface shear stress}

Raupach [24], re-analysed the data given by Marshall [25], and deduced that the form drag is the dominant surface force for rough surfaces with an area density of more than $3 \%$. Most recently, Coceal et al. [26] performed Direct Numerical Simulation (DNS) for flows over 25\% cube arrays (the same surfaces as used in this study) and analysed the vertical profiles of the stress tensor, which consist of viscous, Reynolds and dispersive stress. They found that the viscous term is negligible except on the top surface of the cubes, where its maximum contribution is only about 3\% of the total stress. Furthermore, Snyder and Castro [27] indicated that once the surface is fully aerodynamically rough, which requires the critical roughness Reynolds number $\operatorname{Re}_{c}=\frac{u^{*} z_{0}}{v}>1$ for sharp-edged obstacles, the viscous effects are insignificant. For the four roughnesses and experimental conditions investigated here, $\operatorname{Re}_{\mathrm{c}}$ is at least 15 .

For each roughness, the surface stress $\tau_{p}$, derived from form drag measurements, was used as the baseline of the surface shear stress and compared with the Reynolds shear stress $\tau_{m}$ (here $\tau_{m}=\rho\left(-\overline{u^{\prime} w^{\prime}}\right)$ ), deduced from the spatially averaged shear stress profile within the inertial sublayer over the surface at the same flow conditions. The ratios for all the four surfaces are listed in Table I; the non-dimensional values of the $u^{*}{ }_{(p)} / U_{r}$ derived from $\tau_{p}$ for all the surfaces are also included in Figure 7-10. These data clearly demonstrate that the surface shear stress is in general about 25\% larger than the Reynolds shear stress directly measured in the inertial sublayer. A similar phenomenon was also observed by Iyengar and Farell [28] with a smaller difference of $18 \%$ in their experiments, and they suspected that the discrepancy was due to the cross wire errors in the near wall region where the turbulent intensity was high. Here it is worth mentioning that they used $90^{\circ} \mathrm{x}$-wire in their study. However, in our early work [7], the adequacy of the $120^{\circ} \mathrm{x}$-wire data had been checked and confirmed by the laser Doppler anemometry technique. Therefore, it is believed that the discrepancy between the surface shear stress $\tau_{p}$ and the inertial layer Reynolds shear stress $\tau_{m}$ cannot be attributed to the measurement technique used in this investigation. However, in all cases it is significant that plausible extrapolations of $-\overline{u^{\prime} w^{\prime}}$ from the inertial layer (in which it is not, in fact, constant) to the point 
$(z=d)$ do yield values relatively consistent with the surface stresses implied by the pressure measurements. In any case, deriving a friction velocity from shear stress measurements aloft, which is common practice in wind tunnel simulation of the atmospheric boundary layer, would significantly underestimate the surface friction velocity.

The longitudinal pressure gradient, $d \bar{P} / d x$ (see equation 9 in section 4.4), resulting from boundary layer growth over the rough lower wall and the other three surfaces was estimated. This was found to be consistent with the observed shear stress gradients near the wall. The inevitable consequence is that estimates of the friction velocity from measurements above the roughness sublayer should take the gradient into account in extrapolating down to the zeroplane level. (Table 1 lists a boundary layer depth of about $6 h$, which was about $20 \%$ of the working section depth; the growth rate was estimated as: $d \delta / d x \approx 0.03$.) This argument seems to imply that shear stress profiles in many wind tunnel experiments will be affected by small axial pressure gradients unless either the working section is very deep indeed or the working section roof can be adjusted to keep $U_{r}$ constant.

Reynolds et al. [29] have shown that significant spanwise variations can occur in boundary layers developing over cube arrays, but in this study the surface shear stress $\tau_{p}$ has be derived from the pressure distribution around a single cube, rather than an average of many cubes. As mentioned in Section 2, the total surface drag $\tau_{t}$ was also measured by a floating drag balance. The measurements were compared with $\tau_{p}$ at the same flow condition and the ratio of $\tau_{p} / \tau_{t}$ for a free stream velocity of $10 \mathrm{~m} / \mathrm{s}$ is included in the final row of Table I. One would expect the ratio to be less than unity as $\tau_{t}$ includes both pressure and viscous drag terms. However, it is found that for one surface $\tau_{p}$ is $12 \%$ greater than $\tau_{t}$. A possible explanation could be that the $\tau_{p}$ measurements are based on a single cube whereas $\tau_{t}$ was obtained using the floating drag balance which had many cubes mounted on it, thus giving an average value less susceptible to any spanwise variations that exist in the flow.

Table I Summary of the various layers over the rough surfaces.

\begin{tabular}{|l|l|l|l|l|l|}
\hline & & $\mathrm{C} 20 \mathrm{~S}-25 \%$ & $\mathrm{C} 20 \mathrm{~A}-25 \%$ & $\mathrm{C} 20 \mathrm{~S}-6.25 \%$ & C20A-6.25\% \\
\hline 1 & Boundary layer depth $\delta$ & $6.7 h$ & $6.9 h$ & $6.8 h$ & $6.2 h$ \\
\hline 2 & Top of roughness sublayer & $1.75 h$ & $1.8 h$ & $1.8 h$ & $\sim 4 h$ \\
\hline 3 & $\begin{array}{l}\text { Depth of inertial sublayer }(5 \% \\
\left.\text { variation in }-\overline{u^{\prime} w^{\prime}}\right)\end{array}$ & $1.75 \sim 2.15 h$ & $1.8 \sim 2.4 h$ & $1.8 \sim 2.2 h$ & \\
\hline 4 & $\delta_{\text {inertial sublayer }} \delta$ & $32 \%$ & $35 \%$ & $33 \%$ & \\
\hline 5 & $\begin{array}{l}\text { Vertical region fitted by Log- } \\
\text { law }\end{array}$ & $1.2 \sim 2.75 h$ & $1.2 \sim 2.75 h$ & $1.2 \sim 3 h$ & $1.2 \sim 2.55 h$ \\
\hline 6 & $\begin{array}{l}\text { Equivalent variation in } \\
-u^{\prime} w^{\prime} \% \text { for log-law region }\end{array}$ & $14 \%$ & $10 \%$ & $20 \%$ & \\
\hline 7 & $u^{*}(p) / U_{r}$ & 0.0718 & 0.0682 & 0.0728 & 0.0642 \\
\hline 8 & $\tau_{p} / \tau_{m}$ & 1.26 & 1.22 & 1.32 & \\
\hline 9 & $\tau_{p} / \tau_{t}$ & 1.03 & 0.9 & 1.12 & 0.97 \\
\hline
\end{tabular}




\subsection{Log-law fitting procedure}

In the log-law fitting process described here, the friction velocity is pre-determined from either form drag measurements $u_{(p)}^{*}$ or the shear stress profile $u^{*}{ }_{(m)}$. Assuming that there are $n$ data points in the region, which are selected to fit the logarithmic wind profile, the sum of the leastsquare error for the selected region can be obtained from the difference between the measured velocity and the calculated one from the usual logarithmic law in the following expression:

$$
E=\sum_{i=1}^{n}\left[U_{i}-\frac{u^{*}}{k} \ln \left(\frac{z_{i}-d}{z_{0}}\right)\right]^{2}
$$

where $U_{i}$ is the measured mean velocity at height of $z_{i}$ in the selected region. Both the zeroplane displacement $d$ and the roughness length $z_{0}$ are unknowns, and assigned different values. For each pair of assigned $d$ and $z_{0}$ the total error $E$ was evaluated and the fitting was visually inspected. The values of $d$ and $z_{0}$ which gave a minimum error are the required solution of the log-law fitting process. In this procedure $d$ was constrained to be less than or equal to the height of the roughness elements.

\subsection{Discussions on parameterization}

For each surface, including C20A-6.25\%, the vertical velocity profiles over a repeating unit were spatially averaged. The log-law was then fitted from the lowest measurement point, which was $4 \mathrm{~mm}$ above the cube top, through the roughness sublayer and the inertial sublayer as well as up into the flow above as far as possible, provided that the fitting is visually good (see Figure 12), using $u^{*}(p)$ as the surface friction velocity. The upper limit of the region which was well-fitted by a log-law is also shown in Figure 7-10. The fitting parameters $\left(z_{0}\right.$ and $\left.d\right)$ are listed in the first row of Table II and are used as the baseline when compared with $z_{0}$ and $d$ from other approaches.

The depth of the log-law region is different for different roughnesses and is listed in the $5^{\text {th }}$ row of Table I. The equivalent variation in the spatially averaged shear stress for this region is listed in the $6^{\text {th }}$ row of Table I. For the $25 \%$ surfaces, the log-law region would be consistent with the inertial sublayer defined by the quasi-constant shear stress layer if the latter were allowed about a $10 \%$ variation in $-\overline{u^{\prime} w^{\prime}}$. But for the $6.25 \%$ surfaces, it would require $20 \%$ variation in $-\overline{u^{\prime} w^{\prime}}$ to unify the two approaches indicated by Feddersen et al. [23]. Using one definition of inertial sublayer will not be able to include all the surfaces involved here. It is clear that within the log-law region the shear stress is not constant with height. To explain why, we examine the equation of motion in the longitudinal direction.

Following Finnigan [30] and Coceal and Belcher [31], for ideal steady 2D flow over rough wall, the time and space averaged momentum equation in the stream-wise direction is

$$
\rho\left(\bar{U} \frac{\partial \bar{U}}{\partial x}+\bar{W} \frac{\partial \bar{U}}{\partial z}\right)=-\frac{d \bar{P}}{d x}-\rho\left(\frac{\partial\left\langle\overline{u^{\prime 2}}\right\rangle}{\partial x}+\frac{\partial\left\langle\overline{u^{\prime} w^{\prime}}\right\rangle}{\partial z}\right)-D-\rho\left(\frac{\partial\left\langle\tilde{u}^{2}\right\rangle}{\partial x}+\frac{\partial\langle\tilde{u} \tilde{w}\rangle}{\partial z}\right)
$$

There are new terms on the right hand side. $\left\langle\overline{\left\langle u^{\prime 2}\right.}\right\rangle$ and $\left\langle\overline{u^{\prime} w^{\prime}}\right\rangle$ are the spatially averaged Reynolds stresses, which represent spatially averaged momentum transport due to turbulent 
velocity fluctuations. $\left\langle\tilde{u}^{2}\right\rangle$ and $\langle\tilde{u} \tilde{w}\rangle$ are dispersive stresses, due to momentum transport by the spatial deviations from the time-averaged wind. D is the drag force due to individual roughness elements (and is thus only non-zero within the canopy region). Experimental evidence has shown that, compared with the usual Reynolds stresses, the dispersive stress is negligible in the region above the top of the canopy but may be a larger fraction near the bottom of the canopy. But both stresses tend to be small in the canopy layer where the drag force is important. For fully developed flow where quantities do not change with $x$ under zero pressure gradient conditions, the above equation reduces to

$$
\rho \bar{W} \frac{\partial \bar{U}}{\partial z}=-\rho \frac{\partial \overline{\left\langle u^{\prime} w^{\prime}\right\rangle}}{\partial z}-D-\rho \frac{\partial\langle\tilde{u} \tilde{w}\rangle}{\partial z}
$$

In the log-law layer, $\bar{W}=0$, the convective and dispersive stress terms can be neglected and $D=0$ so that, as in canonical boundary layers, equation (10) becomes simply:

$$
0=-\rho \frac{\partial \overline{\left\langle u^{\prime} w^{\prime}\right\rangle}}{\partial z}
$$

which yields the usual result that $\overline{u^{\prime} w^{\prime}}=$ constant.

For practical flow over a rough wall in a wind tunnel with a fixed roof, as the boundary layer thickness increases with increasing fetch, the cross-sectional area of free stream flow decreases and the pressure gradient along the tunnel is not zero. The dispersive stress and drag terms remain negligible in the region above the top of the canopy so equation (9) becomes:

$$
\rho\left(\bar{U} \frac{\partial \bar{U}}{\partial x}+\bar{W} \frac{\partial \bar{U}}{\partial z}\right)+\frac{d \bar{P}}{d x}+\rho \frac{\partial\left\langle\overline{u^{\prime 2}}\right\rangle}{\partial x}=-\rho \frac{\partial\left\langle\overline{u^{\prime} w^{\prime}}\right\rangle}{\partial z}
$$

Each term was estimated from measurements over a staggered uniform $10 \mathrm{~mm}$ cube arrays with packing density of $25 \%$ at similar conditions as the present study. It seems to show that the first and second terms in the left hand side are not negligible even thought accurate estimation from experimental data is not possible due to the very small values of $\frac{\partial \bar{U}}{\partial x}$ and $\bar{W}$. The axial pressure gradient, determined by measuring free stream velocity at different fetch of the roughness, was found to be the major contributor in the left hand side, and together with other terms roughly balances the estimated shear stress gradient near the wall. This is probably the reason why the shear stress is not constant with height in realistic wind tunnel measurements where the flow never ceases from developing.

In Section 4.1, the definition of the inertial sublayer was arbitrarily chosen by a 5\% variation in the spatially averaged Reynolds shear stress; here we examine the effects of alternative definitions within the log-law region on the surface parameterization while using $u_{(p)}^{*}$ as the friction velocity. The results are listed in Table II. It is found that the roughness length does not vary significantly no matter whether the roughness sublayer is included or not, providing that the velocity profiles in the roughness sublayer are spatially averaged. Furthermore, the roughness length is not sensitive to whether the inertial sublayer is defined by a $5 \%, 10 \%$ or even (in the case of the C20S-6.25\% surface) $20 \%$ variation in the spatially averaged shear stress profiles, as long as it is within the log-law region. 
In Jackson's theory, using the centroid height $H_{c}$ as the zero-plane displacement to fit the same log-law region for each surface would give different $z_{0}$. The results of this procedure are also listed in Table II. For the $6.25 \%$ roughness, the values of $z_{0}$ differ from those obtained by alternative methods by less than the overall uncertainties and the fitting is visually good, as shown in Figure 12. But for the $25 \%$ surfaces which are also displayed in Figure 12, it is not possible to get a reasonably good fit without changing $u^{*}(p)$. This may imply that Jackson's theory works well at low density but breaks down when the flow begins to skim the roughness, at least for cases studied here with an area coverage density of $25 \%$.

As mentioned above, in wind tunnel simulations the friction velocity is often derived from vertical profiles of the shear stress in the inertial sublayer over that surface, which is here denoted as $u_{(m)}^{*}$ to distinguish it from $u_{(p)}^{*} . u^{*}{ }_{(m)}$, derived from the inertial sublayer with 5\% variation in shear stress, was also used as the friction velocity to fit the same log-law region for each surface, as illustrated in Figure 12. The deduced parameters are also included in the final row of Table II. Comparing with the results using $u^{*}(p)$ as the friction velocity, the roughness length derived with $u^{*}{ }_{(m)}$ is consistently and significantly lower than that with $u^{*}{ }_{(p)}$ for the three surfaces analysed here.

Table II Surface properties calculated using $u_{(p)}^{*}$ (except in final row). RS and IS denote the roughness sublayer and inertial sublayer respectively.

\begin{tabular}{|c|c|c|c|c|c|}
\hline Fitting region & $(\mathrm{mm})$ & C20S-25\% & C20A-25\% & C20S-6.25\% & C20A-6.25\% \\
\hline \multirow[t]{2}{*}{ Log-law region } & $z_{0} / h$ & 0.049 & 0.044 & 0.045 & 0.021 \\
\hline & $d / h$ & 0.858 & 0.853 & 0.558 & 0.648 \\
\hline \multirow{2}{*}{$\begin{array}{l}\mathrm{RS} \text { and } \mathrm{IS} \quad(5 \% \\
\text { variation in }-\overline{\left.u^{\prime} w^{\prime}\right)}\end{array}$} & $z_{0} / h$ & 0.052 & 0.046 & 0.047 & \\
\hline & $d / h$ & 0.822 & 0.823 & 0.504 & \\
\hline \multirow{2}{*}{$\begin{array}{l}\text { RS and IS } \frac{(10 \%}{\left.-u^{\prime} w^{\prime}\right)} \\
\text { variation in }\end{array}$} & $z_{0} / h$ & 0.050 & 0.044 & 0.047 & \\
\hline & $d / h$ & 0.845 & 0.853 & 0.504 & \\
\hline \multirow{2}{*}{$\begin{array}{l}\text { RS and IS } \frac{(20 \%}{\left.-w^{\prime}\right)} \\
\text { variation in }\end{array}$} & $z_{0} / h$ & & & 0.045 & \\
\hline & $d / h$ & & & 0.558 & \\
\hline \multirow{2}{*}{$\begin{array}{l}\text { IS } \frac{(5 \%}{} \text { variation } \\
-\overline{\left.u^{\prime} w^{\prime}\right)}\end{array}$} & $z_{0} / h$ & 0.045 & 0.039 & 0.044 & \\
\hline & $d / h$ & 0.964 & 1.000 & 0.618 & \\
\hline \multirow{2}{*}{$\begin{array}{l}\text { IS }(10 \% \text { variation in } \\
-\overline{\left.u^{\prime} w^{\prime}\right)}\end{array}$} & $z_{0} / h$ & 0.043 & 0.038 & 0.044 & \\
\hline & $d / h$ & 1.000 & 1.000 & 0.623 & \\
\hline \multirow{2}{*}{$\begin{array}{l}\text { IS }(20 \% \text { variation in } \\
-\overline{\left.u^{\prime} w^{\prime}\right)}\end{array}$} & $z_{0} / h$ & & & 0.041 & \\
\hline & $d / h$ & & & 0.719 & \\
\hline \multirow{2}{*}{$\begin{array}{l}\text { Log-law region with } \\
d=H_{c}\end{array}$} & $z_{0} / h$ & 0.063 & 0.056 & 0.046 & 0.023 \\
\hline & $H_{c} / h$ & 0.616 & 0.616 & 0.527 & 0.527 \\
\hline \multirow{2}{*}{$\begin{array}{ll}\text { Log-law } & \text { region } \\
\text { using } u^{*}(m) & \\
\end{array}$} & $z_{0} / h$ & 0.031 & 0.029 & 0.024 & \\
\hline & $d / h$ & 0.932 & 0.916 & 0.704 & \\
\hline
\end{tabular}

These data are used to compare with geometrical methods from the literature, such as those of Macdonald et al. [15] and Kastner-Klein and Rotach [17], in Figure 13; both correlations have the correct logical behaviour of the roughness length with variations in area density. The correlation by Macdonald et al. [15] was derived from basic theoretical principles with some simple assumptions; its constants were empirically determined by fitting the experimental data from Hall et al. [32], and the fitting constant in the expression of roughness length is different 
for different roughness patterns, depending on whether an array is staggered or squared. Here it is emphasized that Hall et al. [32] took extensive measurements with pulsed wire anemometry over a series of cube arrays in a wind tunnel at a fetch no more than $22 \mathrm{H}$. Because no direct measurements of the shear stress were made, the three quantities $\left(z_{0}, d\right.$ and $\left.u^{*}\right)$ had to be found by log-law fitting a mean wind profile. Comparing with the subsequent roughness length data of Macdonald et al. [5], where $d$ was estimated by the correlation in Macdonald et al. [15], the scatter is significant. In the experiments of both Hall et al. [32] and Macdonald et al. [5] the fetch was relatively short; it is possible that in both cases the flow does not have a properly developed equilibrium log-law layer. In addition Macdonald et al. [5] noticed that the effective $u^{*}$ derived by fitting the mean wind profile to the log-law with three unknowns of $u^{*}, d$ and $z_{0}$ was consistently $75 \%$ larger than $u^{*}(m)$ determined directly from their shear stress measurements and they were greatly puzzled by the poor agreement between the two methods.

Comparing our data with the prediction by Macdonald et al. [15], the $z_{0}$ values are lower and $d$ larger than their predictions; this may be because the $u_{(p)}^{*}$ in our experiment was independently determined and our fetch $(150 \mathrm{H})$ is significantly larger than theirs $(22 \mathrm{H})$. Also because the velocity profile was spatially averaged over a repeating unit, the roughness length and zeroplane displacement from our experiment should be more representative for the given surface. For comparison purposes, the correlations by Kastner-Klein and Rotach [17] are also included in Figure 13. Our data seem to be closer to the latter than those from Macdonald et al. [15]. However, considering how the correlations were developed, it seems that if the constants were to be obtained by fitting more representative data (i.e. at greater fetch), the correlation by Macdonald et al. [15] would have the potential to perform better.

\section{Conclusions}

Experiments were performed over idealised urban surfaces of different packing densities. The flow at the measurement location has a low $\delta / h$ ratio of $6 \sim 7$. It was found that within the loglaw region the shear stress is not strictly constant with height. The log-law region can be extended to the roughness sublayer provided the velocity profile is spatially averaged. When the three unknowns $\left(u^{*}, d\right.$ and $\left.z_{0}\right)$ are derived from the mean velocity profiles, the final results obtained from the least-square fitting process were sensitive to the initial values being assigned to the unknowns. Therefore, as has been pointed out before, the procedure is somewhat illconditioned and caution should be exercised when using such a method to determine the surface parameters from velocity measurements at a few heights, whether within wind tunnel flows or in the atmospheric boundary layer. The roughness length determined by log-law fitting does not change significantly whether the roughness sublayer was included or not. It has also been shown that the surface shear stress deduced from form drag measurements was consistently larger than the Reynolds shear stress in the log-law region. Therefore, using a friction velocity derived from Reynolds shear stress in the log-law fitting process leads to significant error in the roughness length. The centroid height of the pressure force acting on the roughness element is not always coincident with the zero-plane displacement. Finally, we have shown that at sufficiently low area coverage by the roughness elements, the roughness sublayer can extend over a significant portion of the boundary layer depth, depending on the wind direction. Whether this more extensive spatial inhomogeneity would persist at larger fetches (i.e. at greater $\delta / h$ ) remains on open question. 


\section{Acknowledgement}

We are grateful for the technical support provided by Mr. A. Wells and T. Lawton; without their assistance none of these experiments would have been possible. HC was funded by EPSRC under the grant number GR/R78084/01(P) and PH is funded by NERC under the UWERN programme. Their financial support is greatly appreciated.

\section{References}

[1] Grimmond, C. S. B. and Oke, T. R. (1999), "Aerodynamic properties of urban areas derived from analysis of surface form", Journal of applied meteorology, 38, pp1262-1292.

[2] Petersen, R. L. (1997), "A wind tunnel evaluation of methods for estimating surface roughness length at industrial facilities", Atmospheric Environment, Vol. 31, No. 1, pp45-57.

[3] Bottema, M. (1997), "Urban roughness modelling in relation to pollutant dispersion", Atmospheric Environment, Vol. 31, No. 18, pp3059-3075.

[4] Spanton, A. M., Hall, D. J. and Walker, S. (1996), " A survey of the aerodynamic characteristics of some UK areas", BRE Report CR 177/96, Building Research Establishment, Garston.

[5] Macdonald, R. W., Carter, S. and Slawson, P. R. (2000), "Measurements of mean velocity and turbulence statistics in simple obstacle arrays at 1:200 scale", Thermal fluids report 2000-1, University of Waterloo.

[6] Counihan, J. (1971), "Wind tunnel determination of the rough length as a function of the fetch and the roughness density of three-dimensional roughness elements", Atmospheric Environment, Vol. 5, pp637-642.

[7] Cheng, H. and Castro, I. P. (2002a), "Near wall flow over urban-like roughness", Boundary-Layer Meteorology, 104, pp229-259.

[8] Raupach, M. R., Antonia, R. A. and Rajagopalan, S, (1991), "Rough wall turbulent boundary layers", Applied Mechanical Review, 44, pp1-25.

[9] O'Loughlin, E. M. and Annambhotla, V. S. S. (1969), "Flow phenomena near rough boundaries", Journal of Hydraulic Research, 7, pp231-250.

[10] Mulhearn, P. J. and Finnigan, J. J. (1978), "Turbulent flow over a very rough, random surface", Boundary-Layer Meteorology, 15, pp109-132.

[11] Raupach, M. R., Thom, A. S. and Edwards, I. (1980), "A wind -tunnel study of turbulent flow close to regularly arrayed rough surfaces", Boundary-Layer Meteorology, 18, pp373-397.

[12] Cheng, H. and Castro, I. P. (2002b), "Near-wall flow development after a step change in surface roughness", Boundary-Layer Meteorology, 105, pp411-432.

[13] Oke, T. R. (1987), Boundary layer climates, Routledge.

[14] Jia, Y., Sill, B. L. and Reinhold, T. A. (1998), "Effects of surface roughness element spacing on boundary-layer velocity profile parameters", Journal of Wind Engineering and Industrial Aerodynamics, 73, pp215-230.

[15] Macdonald, R. W., Griffiths, R. F. and Hall, D. J. (1998), "An improved method for the estimation of surface roughness of obstacle arrays", Atmospheric Environment, Vol. 32, No. 11, pp1857-1864.

[16] Jackson, P. S. (1981), "On the displacement height in the logarithmic velocity profile", J. Fluid Mech. Vol. 111, pp15-25.

[17] Kastner-Klein, P. and Rotach, M. (2004), "Mean flow and turbulence characteristics in an urban roughness sublayer", Boundary-Layer Meteorology, 111, pp55-84.

[18] Jiménez, J. (2004), “Turbulent flow over rough walls”, Annu. Rev. Fluid Mech., 36, pp173-196.

[19] Tutu, N. K. and Chevray, R. (1975), "Cross-wire anemometry in high intensity turbulence", $J$. Fluid Mech. 71, pp785-800.

[20] Akins, R. E. and Peterka, J. A. (1977), "Mean force and moment coefficient for buildings in turbulent boundary layer", Journal of Wind Engineering and Industrial Aerodynamics, 2, pp195209. 
[21] Castro, I. P. and Robins, A. G. (1977), "The flow around a surface-mounted cube in uniform and turbulent streams", J. Fluid Mech., Vol. 79, part 2, pp307-335.

[22] Hunt, A. (1982), "Wind-tunnel measurements of surface pressure on cubic building models at several scales", Journal of Wind Engineering and Industrial Aerodynamics, 10, pp137-163.

[23] Feddersen, B., Leitl, B., Rotach, M. W. and Schatzman, M. (2003), "Wind tunnel investigation of the spatial variability of turbulence characteristics in the urban area of Basel city, Switzerland", Proceedings of International Workshop on Physical Modelling of Flow and Dispersion Phenomena, 3-5 September 2003, Prato, Italy, pp23-25.

[24] Raupach, M. R. (1992), "Drag and drag partition on rough surfaces", Boundary-Layer Meteorology, 60, pp375-395.

[25] Marshall, J. K. (1971), "Drag measurements in roughness arrays of varying density and distribution", Agricultural Meteorology, 8, pp269-292.

[26] Coceal, O., Thomas, T. G., Castro, I. P. and Belcher, S. E. (2005), "Numerical simulation of turbulent flow over cubic roughness arrays", submitted to Boundary Layer Meteorol.

[27] Snyder, W. H. and Castro, I. P. (2002), "The critical Reynolds number for rough-wall boundary layer", Journal of Wind Engineering and Industrial Aerodynamics, 90, pp41-54.

[28] Iyengar, A. K. S. and Farell, C. (2001), "Experimental issues in atmospheric boundary layer simulations: roughness length and integral length scale determination", Journal of Wind Engineering and Industrial Aerodynamics, 89, pp1059-1080.

[29] Reynolds, R.T., Hayden, P., Castro, I. P. and Robins, A. G. (2005), "Spanwise variations in nominally two-dimensional rough-wall boundary layers", Submitted to Experiments in Fluids.

[30] Finnigan, J. J. (2000), “Turbulence in plant canopies”, Annu. Rev. Fluid Mech. 32, 519-572.

[31] Coceal, O. and Belcher, S. E. (2004), "A canopy model of mean winds through urban areas", Q.J. R. Meteorol. Soc., 130, pp1349-1372.

[32] Hall, D. J, Macdonald, R., Walker, S. and Spanton, A. M. (1996), "Measurements of dispersion within simulated urban arrays - A small scale wind tunnel study", BRE Client Report, CR178/96. 


\section{Figure List}

Figure 1. Schematic diagram of the rough surfaces used in this study. (a) $6.25 \%$ cube arrays; (b) $25 \%$ cube arrays.

Figure 2. Pressure tapping distribution on the brass cube (solid and open symbols denote the opposite face).

Figure 3. Schematic diagram of the $25 \%$ and $6.25 \%$ staggered surface rafts used to measure the total drag.

Figure 4. The effects of free stream velocity on (a) drag coefficient, (b) friction velocity, (c) friction velocity ratio for staggered and aligned arrays, (d) friction velocity ratio for surfaces of $6.25 \%$ and $25 \%$.

Figure 5. Centroid height of mean drag force on roughness element versus velocity.

Figure 6. Pressure difference coefficient distribution on cubes for different roughness at free stream velocity of $10 \mathrm{~m} / \mathrm{s}$. (a) C20A-6.25\%; (b) C20S-6.25\%; (c) C20A-25\%; (d) C20S-25\%.

Figure 7. Vertical profiles of C20S-25\%. (a) $\bar{U} / U_{r}$; (b) $\overline{u^{\prime 2}} / U_{r}^{2}$; (c) $\overline{w^{\prime 2}} / U_{r}^{2}$; (d) $\overline{u^{\prime} w^{\prime}} / U_{r}^{2}$.

Figure 8. Vertical profiles of C20A-25\%. (a) $\bar{U} / U_{r}$; (b) $\overline{u^{\prime 2}} / U_{r}^{2}$; (c) $\overline{w^{\prime 2}} / U_{r}^{2}$; (d) $\overline{u^{\prime} w^{\prime}} / U_{r}^{2}$.

Figure 9. Vertical profiles of C20S-6.25\%. (a) $\bar{U} / U_{r}$; (b) $\overline{u^{\prime 2}} / U_{r}^{2}$; (c) $\overline{w^{\prime 2}} / U_{r}^{2}$; (d) $\overline{u^{\prime} w^{\prime}} / U_{r}^{2}$.

Figure 10. Vertical profiles of C20A-6.25\%. (a) $\bar{U} / U_{r}$; (b) $\overline{u^{\prime 2}} / U_{r}^{2}$; (c) $\overline{w^{\prime 2}} / U_{r}^{2}$; (d) $\overline{u^{\prime} w^{\prime}} / U_{r}^{2}$.

Figure 11. Vertical profiles of spatially averaged shear stress over a repeating unit for the four surfaces investigated.

Figure 12. Log-law fitting profiles over different surfaces. (a) C20A-25\%; (b) C20S-25\%; (c) C20A-6.25\%; (d) C20S-6.25\%.

Figure 13. Comparing (a) roughness length and (b) zero-plane displacement from our experimental data and the later work by Macdonald et al. [5] with the prediction of geometrical methods developed by Macdonald et al. [15] and Kastner-Klein and Rotach [17] respectively. 